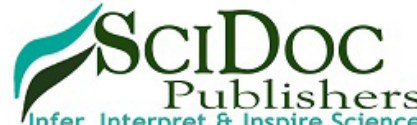

International Journal of Dentistry and Oral Science (IJDOS) ISSN: 2377-8075

\title{
Comparative Evaluation Of Colour Stability Of Two Different Commercially Available Glass Ionomer Cement After Immersion In Carbonated Beverages
}

Research Article

Moulishree Ramesh ${ }^{1}$, Jayalakshmi S², Balaji Ganesh S

${ }^{1}$ Saveetha Dental College and Hospitals, Saveetha Institute of Medical and Technical Sciences (SIMATS), Saveetha University, Chennai- 77, India. ${ }^{2}$ Reader, White lab - Materials Research Centre, Saveetha Dental College and Hospitals, Saveetha Institute of Medical and Technical Sciences [SIMATS], Saveetha University, Chennai- 77, India.

${ }^{3}$ Senior Lecturer, White lab - Materials Research Centre, Saveetha Dental College and Hospitals, Saveetha Institute of Medical and Technical Sciences [SIMATS], Saveetha University, Chennai- 77, India.

Abstract

Background: Glass ionomer cement is a versatile acid base material formed by reaction between weak polymeric acids with glass powdered base. The three essential components of glass ionomer cement namely, water soluble acids like maleic acid or acrylic acid which acts as rate modifier, basic glass which reacts to form salt and water acting as medium, solvent promoting proton release.

Aim: To study the comparative evaluation of two different commercially available glass ionomer cement on immersion in different carbonated beverages.

Materials and Methods: Two different glass ionomer cements were selected, purchased and processed. The samples were prepared as pellets and stored. The samples were then numerically labelled, trimmed and polished. The samples were then immersed in carbonated drinks and left undisturbed for 24 hours. The colour change was then noted using a VITA easy shade advance spectrophotometer. The comparison was documented, analysed using spss statistics version 22.0.

Results: The L,A,B values of the samples were documented, tabulated. The comparison was done using SPSS statistics, independent samples $t$ test. The comparison of delta $\mathrm{E}$ values were done for all samples between immersion of different drinks. On an independent sample test, the $\mathrm{p}$ value was found to be 0.229 , proving to be statistically insignificant, $\mathrm{p}>0.05$.

Conclusion: Pyrax glass ionomer cement showed more colour stability. Sprite drink made the maximum colour change than the other drinks used.

Keywords: Colour Stability; Glass Ionomer Cement; Immersion; Carbonated Beverages; Innovative Technology.

\section{Introduction}

Glass ionomer cement is a versatile acid base material formed by reaction between weak polymeric acids with glass powdered base. The three essential components of glass ionomer cement namely, water soluble acids like maleic acid or acrylic acid which acts as rate modifier, basic glass which reacts to form salt and water acting as medium, solvent promoting proton release. The GIC as a restorative material holds a wide variety of bioactivity which helps adhesion between tooth surfaces increasing the durability. Fluoride release is considered as an important advantage of glass ionomer cement improving oral hygiene over a certain period of time [1]. The clinical performance of glass ionomer cement is determined by various categories and properties like adhesion, fissure sealants, effect over caries dentin, ART, etc. making it ideal for various restorative situations. Glass ionomer cements is well known as a restorative material but with its versatile properties, unique set of advantages and uses, plays a major role in paediatric dentistry in the recent decades as cavity liner, caries control, cavity restorations in primary, permanent tooth, luring cement, etc. [2]. Glass ionomer cement is considered as bioactive material releasing fluoride on $\mathrm{pH}$ acidity, increasing its potential as first choice restorative material in today's world [3].

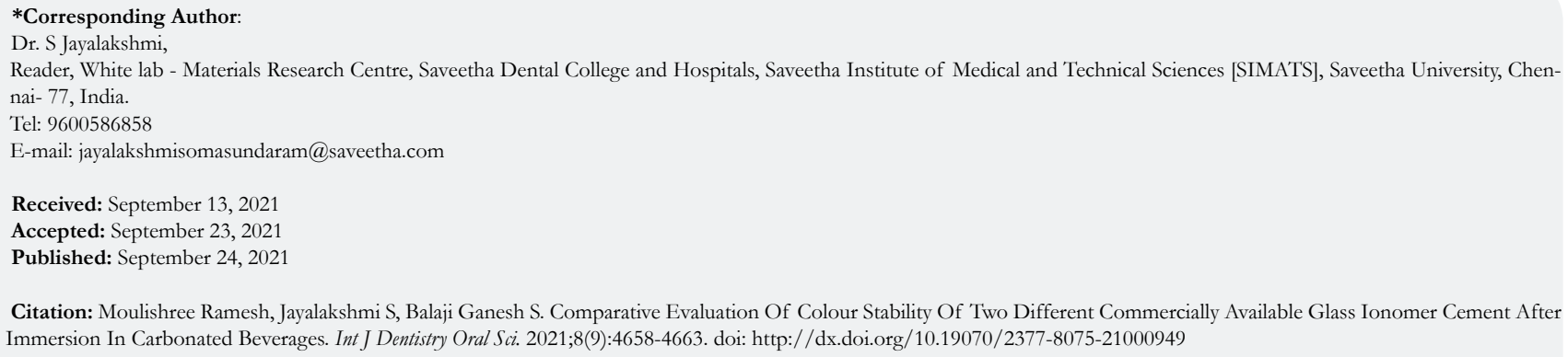

Citation: Moulishree Ramesh, Jayalakshmi S, Balaji Ganesh S. Comparative Evaluation Of Colour Stability Of Two Different Commercially Available Glass Ionomer Cement After Immersion In Carbonated Beverages. Int J Dentistry Oral Sci. 2021;8(9):4658-4663. doi: http://dx.doi.org/10.19070/2377-8075-21000949

Copyright: Dr. S Jayalakshmi ${ }^{\circ} 2021$. This is an open-access article distributed under the terms of the Creative Commons Attribution License, which permits unrestricted use, distribution and reproduction in any medium, provided the original author and source are credited. 
India is a famous place known for its cuisine rich in flavours and spices with high quality food colourants. Knowing the susceptibility of our teeth to various ingredients with high staining capacity in our country, colour stability, the ability to withstand decolourants and maintain the original colour is an important characteristic which needs to be analysed on all categories of restorative materials. Viewing the mechanical and physical properties of the materials, this property is often ignored which should also be included in making a choice in choosing restorative material to be used for the treatment [4]. Understanding the importance of colour stability criteria in restorative dentistry, there are various studies which determine the degree of surface staining of various compounds and colour stability capacity of various restorative materials. Different resin based composites, glass ionomer cement after immersion in various colour stimulants and stains which was measured using spectrophotometer method. Mostly all materials were susceptible with exception of Fuji IX with least susceptibility to stains like coffee, red wine, etc.[5]. In vitro study was done on colour stability of crystallised acetyl resin material on suscepting to different stains and comparing with different restorative materials and was found to be most reliable among the other materials [6]. The colour stability of five different fluoride releasing restorative materials on different $\mathrm{pH}$ levels where glass ionomer cement show great colour stability and no significant changes with $\mathrm{pH}[7]$.

Children generally are more susceptible to sweetened substances, flavours and different coloured drinks. Various restorative materials were exposed to different coloured drinks, stains to check their colour stability. Fuji II showed the most while Fuji IX showed great colour stability after immersion in various beverages. These tests help choose proper restorative material for esthetic reasons. [8]. This study aims to study the comparative evaluation of colour stability of two different commercially available glass ionomer cement after immersion in carbonated beverages.

\section{Materials and Methods}

In this present study, the colour stability of two different commercially available glass ionomer cement after immersion in carbonated beverages. The required amount of glass ionomer ce- ment commonly used - D-tech and pyrax were purchased from the online dental enterprises. The purchased glass ionomer cement was moulded to pellets of $2 \mathrm{~mm}$ diameter. The pellet moulds were then finely trimmed and polished using a micromotor. The pellets were numbered numerically.( figure 1). The colour stability of the samples were calculated and noted before immersion as pre-immersion values. The carbonated drink used in this studycoca cola, sprite, control (distilled water). The sample pellets 1,2( d-tech) , 6,7 (pyrax) were immersed in coca cola drink in a beaker. The sample pellets 3,4( D-tech), 8,9 ( pyrax) were immersed in sprite drink in another beaker. The remaining samples 5 (D-tech) and 10(pyrax) were immersed in control( distilled water). ( figure 2) The sample pellets after immersion in their respective drink were then left undisturbed in a cool, dry place at room temperature. After a period of 24 hours, the samples were taken out, washed and dried. The colour stability of the glass ionomer cement was calculated and noted as post-immersion values. The colour stability was calculated using VITA easyshade spectrophotometer.(figure 3). The pre- immersion and post-immersion values of the sample GIC were tabulated, compared and analysed for comparative evaluation. The statistical analysis was done using SPSS statistics Version 22.0 to study the association and their statistical significance.

\section{Results}

In this present study, the colour stability of glass ionomer cements were focused and the comparative evaluation of different commercially available glass ionomer cements were studied. The L,A,B values of each sample pellet were recorded before( Table 1,2) and after immersion( Table 3,4) in control and carbonated beverages respectively for each sample. The delta $\mathrm{E}$ values of each sample according to their pre-immersion and post-immersion values were calculated( Table 5,6). The delta $E$ values were found to be of moderate colour change values. The comparison of the samples of two different commercially available glass ionomer cement after immersing in different carbonated beverages based on their colour stability was done using independent sample $t$ test analysis in SPSS version 22.0. The association was virtually represented in table 5. The delta $\mathrm{E}$ value showed the range of colour

Figure 1. The picture representing the sample pellets of two different types of commercially available glass ionomer cement in which the first five( numbered- 1, 2, 3, 4, 5) were from Dtech and the next five (numbered- 6, 7, 8, 9, 10) were from Pyrax was used for this present study.

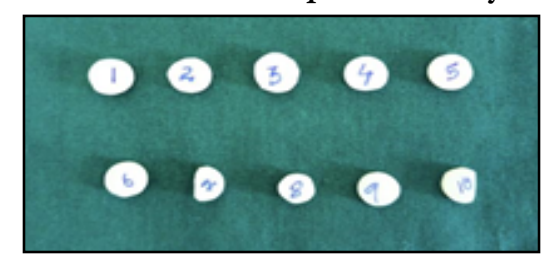

Figure 2. The picture represents the selected GIC sample pellets numbered for our convenience kept near the carbonated beverages in which the two different GIC is to be immersed to compare their colour stability. 1,2,6,7 to be immersed in cocacola, $3,4,8,9$ to be immersed in sprite drink whereas the remaining 5,10 to be immersed in distilled water as control.

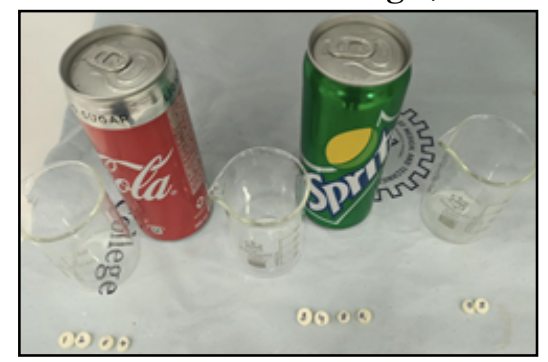


Figure 3. The picture represents the recording of L,A,B values of each sample pellet used before and after immersion in carbonated beverages and entered as pre immersion and post immersion values for comparative study using VITA easyshade spectrophotometer.

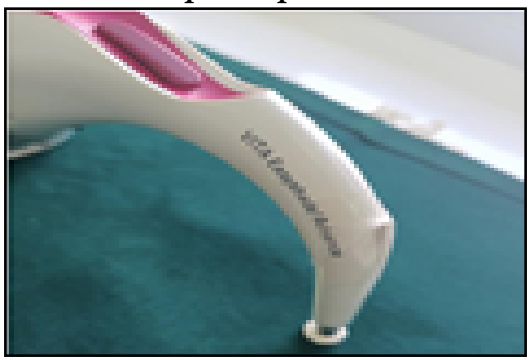

Table 1. Table representing the L,A,B values collected for the D-Tech samples before immersion using VITA easyshade spectrophotometer.

\begin{tabular}{|c|c|c|c|}
\hline SAMPLE NUMBER & L VALUE & A VALUE & B VALUE \\
\hline 1 & 78.1 & 3.2 & 28.7 \\
\hline 2 & 74.4 & 2.7 & 24.2 \\
\hline 3 & 73 & 3.2 & 24.5 \\
\hline 4 & 82.4 & 2 & 22.7 \\
\hline 5 & 81.9 & 2.8 & 26.8 \\
\hline
\end{tabular}

Table 2. Table represents the L,A,B values collected for Pyrax sample before immersion using VITA easyshade spectrophotometer.

\begin{tabular}{|c|c|c|c|}
\hline SAMPLE NUMBER & L VALUE & A VALUE & B VALUE \\
\hline 6 & 88.8 & 0.7 & 21.2 \\
\hline 7 & 87.6 & 0.3 & 21.8 \\
\hline 8 & 89.1 & 1.9 & 21.6 \\
\hline 9 & 88.6 & 0.7 & 23.4 \\
\hline 10 & 88.9 & 0 & 19.1 \\
\hline
\end{tabular}

Table 3. Table representing the L,A,B values of D-Tech samples collected after immersion using VITA easyshade photospectrometer.

\begin{tabular}{|c|c|c|c|}
\hline SAMPLE NUMBER & L VALUE & A VALUE & B VALUE \\
\hline 1 & 76.4 & 2.5 & 25.5 \\
\hline 2 & 75.3 & 2.4 & 24.8 \\
\hline 3 & 73.7 & 3.4 & 30.4 \\
\hline 4 & 87.2 & 1.4 & 23.5 \\
\hline 5 & 78.3 & 1.2 & 19.4 \\
\hline
\end{tabular}

Table 4. Table showing the L,A,B values of the pyrax samples collected after immersion using VITA easyshade spectrophotometer.

\begin{tabular}{|c|c|c|c|}
\hline SAMPLE NUMBER & L VALUE & A VALUE & B VALUE \\
\hline 6 & 87.1 & 1.8 & 23.7 \\
\hline 7 & 88.4 & 1.2 & 23.8 \\
\hline 8 & 91.4 & 1.8 & 20.4 \\
\hline 9 & 93.3 & 0.1 & 20.4 \\
\hline 10 & 85.9 & 0.4 & 20.7 \\
\hline
\end{tabular}

Table 5. Table showing the delta $E$ values of the D-Tech samples used in this study.

\begin{tabular}{|c|c|}
\hline S. No & Delta E value \\
\hline 1 & 3.6905 \\
\hline 2 & 1.0296 \\
\hline 3 & 5.9447 \\
\hline 4 & 4.9031 \\
\hline 5 & 8.3833 \\
\hline
\end{tabular}


Table 6. Table showing the delta $E$ values of the pyrax samples used in this present study.

\begin{tabular}{|c|c|}
\hline S. No & Delta E value \\
\hline 1 & 3.2171 \\
\hline 2 & 2.3345 \\
\hline 3 & 2.5962 \\
\hline 4 & 5.6080 \\
\hline 5 & 3.4234 \\
\hline
\end{tabular}

Table 7. Table representing the mean average, standard deviation and significance for the two different samples of GIC.

\begin{tabular}{|c|c|c|c|c|c|}
\hline \multicolumn{5}{|c|}{ Group Statistics } \\
\hline \multicolumn{2}{|c|}{ Groups } & N & Mean & Std. Deviation & significance \\
\hline \multirow{2}{*}{ Delta E } & D-tech & 5 & 4.790240 & 2.7206358 & 0.229 \\
\cline { 2 - 6 } & Pyrax & 5 & 3.435840 & 1.2926935 & \\
\hline
\end{tabular}

Figure 4. Bar graph representing the comparison of mean delta E values of two different commercially available glass ionomer cement samples. $X$ axis represents glass ionomer cement sample type whereas $Y$ axis represents the mean value of the delta $E$ values. The mean delta $E$ value of $D$-Tech was found to be 4.790; pyrax was found to be 3.435 for the calculated delta $E$ values from pre-immersion and post-immersion values. The comparison was found to be statistically insignificant.( independent sample $t$ test; $\mathrm{p}=0.229 ; \mathrm{p}<0.05)$.

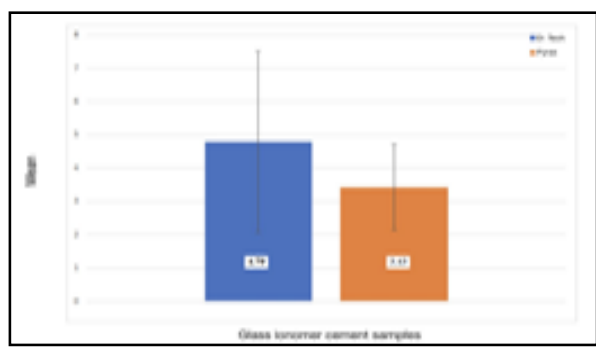

change seen in the sample after immersion. The group statistics such as mean, standard deviation, mean standard error was added to the table, (Table 7) based on the delta E values derived. Figure 4, the bar graph was derived from the comparative evaluation done via sample $t$ test showing the mean average of delta $E$ values from pre-immersion and post-immersion values of different GIC samples.

\section{Discussion}

Our team has extensive knowledge and research experience that has translated into high quality publications [9-28]. In the present study, the colour stability of the two different commercially available glass ionomer cements were studied via $L, A, B$ values by comparing the pre-immersion and post-immersion values. The L,A,B values of d-tech, pyrax samples were noted before and after immersing in the carbonated drinks- coca cola, sprite with control as pre-immersion and post-immersion values respectively. The delta-E values of each sample were calculated and tabulated. On comparing the colour stability of the samples between the different types of carbonated drink on the basis of the delta-E values of the samples, it was found that the colour variation was more on immersion in sprite drink. On comparing the colour stability between the glass ionomer cement brands- D-Tech, Pyrax, the delta $E$ values were maximum in d-tech in comparison with pyrax showing that pyrax have more colour stability than D-Tech. The delta $\mathrm{E}$ difference seen in the correlation graph is less than 10 which shows that the colour change in the cement seems to be moderate with not much colour change and significant po- tential of colour stability in both the GIC brands. On immersion in coca cola, the colour change was more in pyrax showing 2.78 delta $\mathrm{E}$ difference while D-Tech showed 2.38 delta $\mathrm{E}$ difference. On immersion in sprite drink, D-Tech showed a greater colour change than pyrax with delta $\mathrm{E}$ value 5.42 while pyrax had 4.10 delta $\mathrm{E}$ difference. Dtech showed maximum colour change in control with 5.9 deltaE value while pyrax showed no colour change. These evaluations help us conclude that D-tech seems to show maximum colour change than pyrax which inturn shows that pyrax has more colour stability than D-tech. On comparing the colour change based on the carbonated drink, sprite showed maximum colour change than coca-cola.

In this present study, the comparison between the colour stability of different GIC after immersion in different carbonated beverages were done via delta $\mathrm{E}$ values obtained from the L,A,B values of all the sample pellets. The delta $E$ values of both the carbonated beverages and control was correlated and compared between two different commercially available GIC used. The statistical significance of this correlation and delta $\mathrm{E}$ values were studied using independent sample t test where the $\mathrm{p}$-value was found to be $0.229, \mathrm{p}<0.05$. the limitations like less sample pellets, short trial period, comparison of only two commercial GICs should be minimised and focus on enhancing the statistical significance of the study.

Previously, there were various studies done focusing on the colour stability of several adhesive materials exposed to different adverse environmental conditions. The effect of thermocycling on the 
colour stability of adhesives like Cention N, Fuji IX GP Extra, and Fuji IX GP using spectrophotometer. It was found that there exists significant colour change on thermocycling which was statistically significant [29]. Previously, The effect of different types of children's beverages on the colour stability of different types of GIC- giomer, resin-modified glass ionomer cement was focused. The glass ionomer on immersion in beverages like juice, bournvita, coke, the giomer cement showed less colour change with better colour stability [30]. The colour stability of different restorative materials- conventional GIC, composite, porcelain on immersion in red wine was studied. The colour change was noted and statistically analysed via an independent sample t test and found that porcelain had higher colour stability than GIC and composite [31]. These studies help us understand the importance of colour stability, their effect on immersion at different mediums and exposure to various conditions.

In the present study, the effect of carbonated drink over glass ionomer cements were studied, the colour changes analysed using delta $\mathrm{E}$ values via spectrophotometer. The correlation between different carbonated drinks via delta $\mathrm{E}$ values were studied. The present study holds certain limitations like limited sample size, the comparative evaluation was done only between two different brands over a limited trial period. In future studies, more inclusion criterias should be included and compared with many more GIC brands over a long trial period.

\section{Conclusion}

On comparative evaluation, Pyrax glass ionomer cement showed maximum colour stability with limited colour change. Sprite drink showed maximum colour change in GIC in comparison with coca cola and control (distilled water). Further studies should be done to see the colour stability on various restorative materials and by different drinks and spread awareness about the same.

\section{Acknowledgement}

This research was done under the supervision of the Department of Research of Saveetha Dental College and Hospitals. We sincerely show gratitude to the corresponding guides who provided insight and expertise that greatly assisted the research.

\section{Source of Funding}

The present study was supported by

- Saveetha Dental College and Hospitals, Saveetha Institute of Medical and Technical Sciences, Saveetha University, Chennai

- Sarkov health services, chennai.

\section{References}

[1]. Sidhu SK, Nicholson JW. A review of glass-ionomer cements for clinical dentistry. J. Funct. Biomater. 2016 Sep;7(3):16.

[2]. Specification for dental glass ionomer cements.

[3]. Davidson CL. Advances in glass-ionomer cements. J Appl Oral Sci. 2006;14(SPE):3-9

[4]. Samra AP, Pereira SK, Delgado LC, Borges CP. Color stability evaluation of aesthetic restorative materials. Braz Oral Res. 2008 Sep;22(3):205-10.

[5]. Bagheri R, Burrow MF, Tyas M. Influence of food-simulating solutions and surface finish on susceptibility to staining of aesthetic restorative materials. J
Dent. 2005 May;33(5):389-98.Pubmed PMID: 15833394.

[6]. Aburaisi S, Basha A, Al Najjar K, Al Saqat H, Al Askar F, Al Nazer F. The colour stability of crystallized acetyl resin material in comparison to other restorative materials. An in-vitro study. BDJ Open. 2021 Feb 24;7(1):11. Pubmed PMID: 33627634.

[7]. Imparato JC, Garcia A, Bonifácio CC, Scheidt L, Raggio DP, Mendes FM, et al. Color stability of esthetic ion-releasing restorative materials subjected to $\mathrm{pH}$ variations. J Dent Child (Chic). 2007 Sep-Dec;74(3):189-93.Pubmed PMID: 18482512

[8]. Tunc ES, Bayrak S, Guler AU, Tuloglu N. The effects of children's drinks on the color stability of various restorative materials. J Clin Pediatr Dent. 2009 Dec 1;34(2):147-50

[9]. Muthukrishnan L. Imminent antimicrobial bioink deploying cellulose, alginate, EPS and synthetic polymers for 3D bioprinting of tissue constructs. Carbohydr Polym. 2021 May 15;260:117774.Pubmed PMID: 33712131.

[10]. PradeepKumar AR, Shemesh H, Nivedhitha MS, Hashir MMJ, Arockiam S, Uma Maheswari TN, et al. Diagnosis of Vertical Root Fractures by Conebeam Computed Tomography in Root-filled Teeth with Confirmation by Direct Visualization: A Systematic Review and Meta-Analysis. J Endod. 2021 Aug;47(8):1198-1214.Pubmed PMID: 33984375.

[11]. Chakraborty T, Jamal RF, Battineni G, Teja KV, Marto CM, Spagnuolo G. A Review of Prolonged Post-COVID-19 Symptoms and Their Implications on Dental Management. Int J Environ Res Public Health. 2021 May 12;18(10):5131.Pubmed PMID: 34066174.

[12]. Muthukrishnan L. Nanotechnology for cleaner leather production: a review. Environ Chem Lett. 2021 Jan 13;19(3):2527-49.

[13]. Teja KV, Ramesh S. Is a filled lateral canal - A sign of superiority? J Dent Sci. 2020 Dec;15(4):562-563.Pubmed PMID: 33505634.

[14]. Narendran K, MS N, SARVANAN A, SUKUMAR E. Synthesis, Characterization, Free Radical Scavenging and Cytotoxic Activities of Phenylvilangin, a Substituted Dimer of Embelin. Indian J. Pharm. Sci. 2020 Sep 1;82(5).

[15]. Reddy P, Krithikadatta J, Srinivasan V, Raghu S, Velumurugan N. Dental Caries Profile and Associated Risk Factors Among Adolescent School Children in an Urban South-Indian City. Oral Health Prev Dent. 2020 Apr 1;18(1):379-386.Pubmed PMID: 32618460.

[16]. Sawant K, Pawar AM, Banga KS, Machado R, Karobari MI, Marya A, et al. Dentinal Microcracks after Root Canal Instrumentation Using Instruments Manufactured with Different NiTi Alloys and the SAF System: A Systematic Review. Appl. Sci. 2021 Jan;11(11):4984.

[17]. Bhavikatti SK, Karobari MI, Zainuddin SLA, Marya A, Nadaf SJ, Sawant VJ, et al. Investigating the Antioxidant and Cytocompatibility of Mimusops elengi Linn Extract over Human Gingival Fibroblast Cells. Int J Environ Res Public Health. 2021 Jul 4;18(13):7162.Pubmed PMID: 34281099.

[18]. Karobari MI, Basheer SN, Sayed FR, Shaikh S, Agwan MAS, Marya A, et al. An In Vitro Stereomicroscopic Evaluation of Bioactivity between Neo MTA Plus, Pro Root MTA, BIODENTINE \& Glass Ionomer Cement Using Dye Penetration Method. Materials (Basel). 2021 Jun 8;14(12):3159.Pubmed PMID: 34201321.

[19]. Rohit Singh T, Ezhilarasan D. Ethanolic Extract of Lagerstroemia Speciosa (L.) Pers., Induces Apoptosis and Cell Cycle Arrest in HepG2 Cells. Nutr Cancer. 2020;72(1):146-156.Pubmed PMID: 31149840.

[20]. Ezhilarasan D. MicroRNA interplay between hepatic stellate cell quiescence and activation. Eur J Pharmacol. 2020 Oct 15;885:173507.Pubmed PMID: 32858048.

[21]. Romera A, Peredpaya S, Shparyk Y, Bondarenko I, Bariani GM, Abdalla $\mathrm{KC}$, et al. Bevacizumab biosimilar BEVZ92 versus reference bevacizumab in combination with FOLFOX or FOLFIRI as first-line treatment for metastatic colorectal cancer: a multicentre, open-label, randomised controlled trial. The Lancet Gastroenterol Hepatol. 2018 Dec 1;3(12):845-55.

[22]. Raj R K, D E, S R. 囚-Sitosterol-assisted silver nanoparticles activates Nrf2 and triggers mitochondrial apoptosis via oxidative stress in human hepatocellular cancer cell line. J Biomed Mater Res A. 2020 Sep;108(9):18991908.Pubmed PMID: 32319188.

[23]. Vijayashree Priyadharsini J. In silico validation of the non-antibiotic drugs acetaminophen and ibuprofen as antibacterial agents against red complex pathogens. J Periodontol. 2019 Dec;90(12):1441-1448.Pubmed PMID: 31257588.

[24]. Vijayashree Priyadharsini J, Smiline Girija AS, Paramasivam A. In silico analysis of virulence genes in an emerging dental pathogen A. baumannii and related species. Arch Oral Biol. 2018 Oct;94:93-98.Pubmed PMID: 30015217.

[25]. Uma Maheswari TN, Nivedhitha MS, Ramani P. Expression profile of salivary micro RNA-21 and 31 in oral potentially malignant disorders. Braz Oral Res. 2020 Feb 10;34:e002.Pubmed PMID: 32049107.

[26]. Gudipaneni RK, Alam MK, Patil SR, Karobari MI. Measurement of the Maximum Occlusal Bite Force and its Relation to the Caries Spectrum of First Permanent Molars in Early Permanent Dentition. J Clin Pediatr Dent. 
2020 Dec 1;44(6):423-428.Pubmed PMID: 33378468.

[27]. Chaturvedula BB, Muthukrishnan A, Bhuvaraghan A, Sandler J, Thiruvenkatachari $\mathrm{B}$. Dens invaginatus: a review and orthodontic implications. $\mathrm{Br}$ Dent J. 2021 Mar;230(6):345-50.

[28]. Kanniah P, Radhamani J, Chelliah P, Muthusamy N, Joshua Jebasingh Sathiya Balasingh Thangapandi E, Reeta Thangapandi J, et al. Green synthesis of multifaceted silver nanoparticles using the flower extract of Aerva lanata and evaluation of its biological and environmental applications. ChemistrySelect. $2020 \mathrm{Feb} 21 ; 5(7): 2322-31$.

[29]. Bhattacharya S, Purayil TP, Ginjupalli K, Kini S, Pai S. Effect of thermo- cycling on the colour stability of aesthetic restorative materials: An in-vitro spectrophotometric analysis. Pesqui Bras Odontopediatria Clin Integr . 2020;20.

[30]. Hotwani K, Thosar N, Baliga S. Comparative in vitro assessment of color stability of hybrid esthetic restorative materials against various children's beverages. J Conserv Dent. 2014 Jan;17(1):70-4.Pubmed PMID: 24554866.

[31]. Chakravarthy Y, Clarence S. The effect of red wine on colour stability of three different types of esthetic restorative materials: An in vitro study. J Conserv Dent. 2018 May-Jun;21(3):319-323.Pubmed PMID: 29899637. 